\title{
Influence of Contact Angle and Tube Size on Capillary-Driven Flow Under Microgravity
}

\author{
Caixia Wang, Shenghua Xu, Zhiwei Sun, $*$ and Wenrui Hu \\ Chinese Academy of Sciences, 100190 Beijing, People's Republic of China
}

DOI: $\underline{10.2514 / 1.41899}$

\begin{abstract}
The influence of contact angle and tube radius on the capillary-driven flow for circular cylindrical tubes is studied systematically by microgravity experiments using the drop tower. Experimental results show that the velocity of the capillary flow decreases monotonically with an increase in the contact angle. However, the time-evolution of the velocity of the capillary flow is different for different sized tubes. At the beginning of the microgravity period, the capillary flow in a thinner tube moves faster than that in a thicker tube, and then the latter overtakes the former. Therefore, there is an intersection between the curves of meniscus velocity vs microgravity time for two differently sized tubes. In addition, for two given sized tubes this intersection is delayed when the contact angle increases. The experimental results are analyzed theoretically and also supported by numerical computations.
\end{abstract}

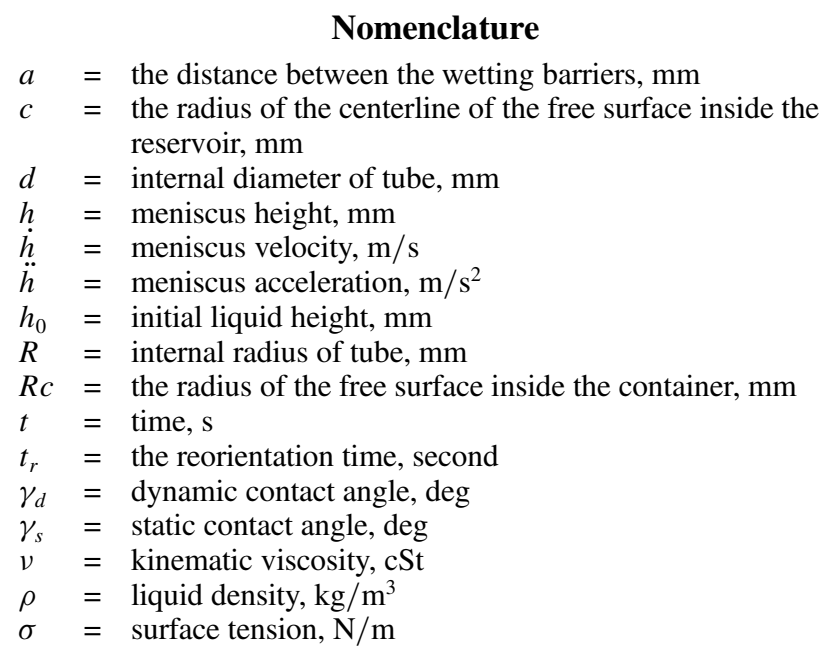

\section{Introduction}

$\mathbf{T}$ HE capillary-driven flow has long been investigated by scientists and engineers [1-3] both theoretically and experimentally, because of its importance for fluid dynamics, surface science, and especially for fluid management in space. In earlier years, most relevant experiments were performed under normal gravity conditions $[\underline{4}, \underline{5}]$. However, the capillary-driven flow becomes remarkable only when the size of the capillary tube is small for terrestrial experiments. To solve this problem, many researchers recently began to experimentally study the capillary-driven flow under microgravity because large sized capillary tubes can be used by removing the effect of gravity. Related theories are also progressing. Levine et al. [6] made modifications to the Lucas-Washburn equation $[1,2]$ by introducing the appropriate momentum balance equations and presented the most detailed theory for the capillary rise in tubes. Dreyer et al. [7] examined the capillary-driven flow under microgravity in a $4.7 \mathrm{~s}$ drop tower using parallel plate channels, and the results showed that at an early stage of penetration, the capillary rise is governed by inertial forces. Weislogel and Lichter [8] studied

Received 29 October 2008; revision received 25 June 2009; accepted for publication 25 June 2009. Copyright (C) 2009 by the American Institute of Aeronautics and Astronautics, Inc. All rights reserved. Copies of this paper may be made for personal or internal use, on condition that the copier pay the $\$ 10.00$ per-copy fee to the Copyright Clearance Center, Inc., 222 Rosewood Drive, Danvers, MA 01923; include the code 0001-1452/09 and \$10.00 in correspondence with the CCC.

*Key Laboratory of Microgravity, National Microgravity Laboratory, Institute of Mechanics; sunzw@imech.ac.cn. the capillary-driven flow along the interior corners of a partly filled container in microgravity using a $2.2 \mathrm{~s}$ drop tower and modified $h-t$ (rising height vs time) dependencies were observed. Chen and Collicott [9] presented the experimental results on capillary flow in a vane-wall gap geometry obtained with the Purdue drop tower, and effects of geometric parameters, contact angle, and the fluid viscosity on the flow were investigated. An experimental study on the capillary-driven flow and the final equilibrium position of the interface in double proboscis containers conceived and developed by Concus et al. [10] was carried out by the USML-2 space shuttle flight. For circular cylinders, the capillary rise flow was extensively studied by Stange et al. [11] and typical three-flow regimes were identified with the aid of analytical and experimental results. Dreyer has included some recent progress in the study of capillary flow under microgravity in his book [12].

Because of removal of the ubiquitous effects of gravity in terrestrial experiments on fluid behavior, experiments in microgravity make it possible to deepen our knowledge of the nature of capillary-driven flow. This knowledge is particularly important for the design of fluid transfer systems of spacecraft, such as propellant and cryogenic fluid tanks, thermal control systems, coolant reservoirs, and systems for collection, storage, and provision of water [13]. It is also helpful in improving modeling studies predicting fluid flows in porous media $[14,15]$. Under microgravity conditions, the flow behavior of fluid is rather complicated due to the competition of different influencing factors, including contact angle, tube size, and so forth. However, there is still a lack of systematic study on the effects of these factors on the dynamic of capillary-driven flow.

In this study we performed a series of experiments in a Beijing drop tower, which provided $3.6 \mathrm{~s}$ of microgravity, to systematically study the influence of contact angle and tube radius on the capillarydriven flow under microgravity. As a basic model of fluid transfer systems, circular cylindrical tubes are adopted in the experiments. The results show that the larger contact angle will decrease the velocity of the capillary flow. We found, however, that the time evolvements of the velocity of the capillary flow are different for different sized tubes. At the beginning of the microgravity period, the capillary flow in a thinner tube moves faster than in a thicker one, and then the latter overtakes and outstrips the former. Therefore, there is an intersection between the curves of meniscus velocity vs microgravity time for two different sized tubes. In addition, for two given sized tubes this intersection delays when the contact angle becomes larger. The experimental results are analyzed theoretically and also supported by relevant numerical computations.

\section{Experimental Setup and Procedure}

A cylindrical acrylic container with internal diameter $150 \mathrm{~mm}$ and total height $150 \mathrm{~mm}$ was used for the experiments. The container was 


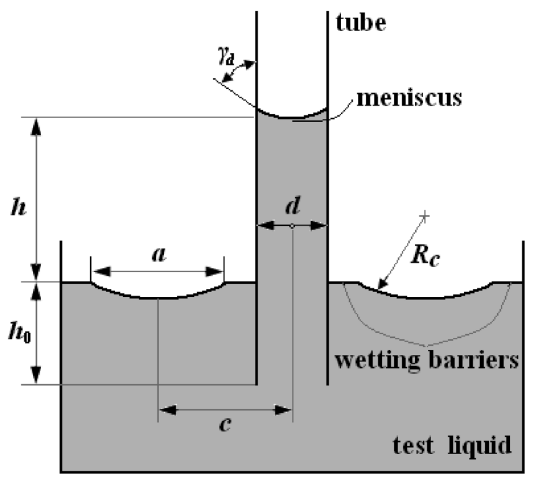

Fig. 1 Schematic representation of the geometry of experimental apparatus for measuring the meniscus height. The initial liquid height is named $h_{0}$ (=depth of immersion), the meniscus height $h$. The tube diameter is $d$, the dynamic contact angle is $\gamma_{d}$. The radius of the free surface inside the container $R c$ is calculated with the distance $a$ between the wetting barriers and the radius $c$ of the centerline of the free surface inside the reservoir.

filled with liquid up to a level of $60 \mathrm{~mm}$. The tubes, manufactured from acrylic plastic and open on the bottom and top, were immersed in the liquid from above (see Fig. 1). Internal diameters of $d=4,6$, and $10 \mathrm{~mm}$ were used. The immersion depth of the tubes (equals initial liquid height $h_{0}$ ) was $h_{0}=10 \mathrm{~mm}$. A charge-coupled device (CCD) camera and a Hi8 video recorder system attached at the outer sides of the tubes were used to monitor and record the position of the meniscus. Wetting barriers were used to prevent the liquid from climbing up the outer side of the tubes and the inner container wall.

Because distilled water and ethanol produce equilibrium contact angles on acrylic 80 and 0 deg, respectively, the two fluids can be mixed in different proportions to produce a range of contact angles. Three aqueous ethanol mixtures were used: $\mathrm{Eth} / \mathrm{H}_{2} \mathrm{O} 20 / 80$ (\%vol.), Eth $/ \mathrm{H}_{2} \mathrm{O}$ 30/70(\%vol.), and Eth $/ \mathrm{H}_{2} \mathrm{O}$ 40/60(\%vol.). Similar materials have been used by Weislogel and Ross [16] to adjust the contact angles in experiments.

The surface tensions, viscosities, and densities of these aqueous ethanol solutions were measured specifically for this research work using standard laboratory procedures and are shown in Table 1 with their respective uncertainties. The equilibrium contact angles for each fluid/solid pair were measured by an optical contact angle measuring device (OCA20) with the software for contact angle measurements (SCA) software. This SCA software is based on goniometry, and contact angle can be assessed directly by measuring the angle formed between the solid and the tangent to the drop surface. The assignment of the tangent line that will define the contact angle is a factor that can limit the reproducibility of contact angle measurements. Conventional goniometry relies on the consistency of the operator in the assignment of the tangent line. This can lead to significant error, especially subjective error between multiple users. SCA software overcomes this problem by using computer analysis of the drop shape to generate consistent contact angle data. Equilibrium contact angles determined in this approach are also listed in Table 1. The properties of experimental fluids were measured at room temperature $\left(25^{\circ} \mathrm{C}\right)$.

Because the contact angles are strongly affected by surface contamination, a procedure for cleaning the tubes is indispensable. Before each experiment, the tubes were rinsed twice with ethanol and cleaned ultrasonically in a warmed detergent for $30 \mathrm{~min}$. After the cleaning, the tubes were again rinsed with the respective test liquid several times and allowed to air dry. Through these cleaning procedures, the surface conditions of the tubes were sufficient to make the resultant data repeatable.

A spacecraft in orbit can provide a long and stable microgravity environment but it is very costly. Alternatively, the drop tower is a cost-efficient way to provide short term microgravity at low cost. In this study the experiments were conducted in the dual-drop capsule system of the drop tower in Beijing. This facility provides $3.6 \mathrm{~s}$ of free fall with $g$-residual estimated at $10^{-5} g_{0}$ [17]. After the release of the drop capsule, the weightlessness condition is reached within a few milliseconds. The liquid surface inside the tube changes from the $1 g_{0}$ shape (flat at the tube center and curved in the vicinity of the liquidsolid contact line) to the spherical microgravity shape. At the same time, the liquid starts to flow into the tube.

Figure $\underline{2}$ shows a typical image recorded from the CCD camera. The internal diameter of the tube was $d=6 \mathrm{~mm}$ and the initial liquid height $h_{0}=10 \mathrm{~mm}$ and the liquid was Eth $/ \mathrm{H}_{2} \mathrm{O} 30 / 70$ (\%vol.). The first picture $(t=0)$ shows the $1 g_{0}$ fluid configuration before the drop of the capsule. With the release of the drop capsule, a microgravity condition is established and the liquid starts to flow into the tube. The progression of the meniscus height $h$ with experiment time $t$ was evaluated from the recorded video data. By varying contact angle and tube size, data of a series of progression of $h \sim t$ was achieved.

\section{Results and Discussion}

\section{A. Experimental Results}

The results of the drop-tower tests are presented in Figs. 3 and 4. Figure 3 shows typical experimental results of the effect of contact angle on the capillary-driven flow under microgravity conditions, where meniscus height $h$ is plotted vs microgravity time $t$. Stange et al. [11] predicted and verified that for the capillary rise in circular tubes there exists three successive stages in which the meniscus height is dominated by the inertia force, convective losses, and viscous forces acting against the driving capillary force, respectively. As a result of the three different regions, the meniscus height increases proportional to the square of time $\left(h \sim t^{2}\right)$, then a linear dependence $(h \sim t)$, and finally the Lucas-Washburn behavior $(h \sim \sqrt{t})$.

For the case of $d=4 \mathrm{~mm}$ (Fig. 3a) in our experiments, the flow enters the Lucas-Washburn region $\overline{(h} \sim \sqrt{t})$ very rapidly for three different contact angles. From Fig. 3a, it can be seen that the slope of all $4 \mathrm{~mm}$ curves decreases almost immediately after the start of the meniscus rise. Figure 3 a also shows that the smaller the contact angle, the faster the time for the flow to enter the Lucas-Washburn region. In the case of $d=6 \mathrm{~mm}$ (Fig. $3 \mathrm{~b}$ ) for the contact angle $\gamma_{s}=42 \mathrm{deg}$ the curve entered the Lucas-Washburn flow $(h \sim \sqrt{t})$ before $3.6 \mathrm{~s}$, whereas for the contact angles $\gamma_{s}=54 \mathrm{deg}$ and $\gamma_{s}=66 \mathrm{deg}$ the flow did not. In the case of $d=10 \mathrm{~mm}$ (Fig. 3c), all three curves are in the first region $\left(h \sim t^{2}\right)$ before the end of the $3.6 \mathrm{~s}$ microgravity time. However, all three figures in Fig. 3 show that the meniscus height $h$ decreased with the increase of contact angle during microgravity duration for all cases of tube radii, despite differences in the time to enter Lucas-Washburn flow.

Figure 4 shows typical experimental results of the effect of tube size on the capillary-driven flow under microgravity conditions, where meniscus height $h$ is plotted vs microgravity time $t$. In the case of $\gamma_{s}=66 \mathrm{deg}$ (Fig. 4a), for $d=4 \mathrm{~mm}$, the curve covers the complete process from the initial meniscus development to the final Lucas-Washburn behavior $(h \sim \sqrt{t})$, whereas for $d=6 \mathrm{~mm}$ and $d=10 \mathrm{~mm}$ the curves remain in the first region $\left(h \sim t^{2}\right)$ until the end of $3.6 \mathrm{~s}$ microgravity. During the $3.6 \mathrm{~s}$ microgravity, the meniscus heights increased with the decrease of tube size.

Table 1 Relevant fluid/interfacial properties

\begin{tabular}{lcccc}
\hline \hline & $\sigma \pm 5 \%, \mathrm{~N} / \mathrm{m}$ & $\rho \pm 5 \%, \mathrm{~kg} / \mathrm{m}^{3}$ & $v \pm 1 \%, \mathrm{cSt}$ & $\gamma_{s} \pm 2, \mathrm{deg}$ \\
\hline Eth/ $\mathrm{H}_{2} \mathrm{O} 20 / 80$ (\%vol.) & 0.0443 & 958 & 1.49 & $66 \mathrm{deg}$ \\
Eth/ $\mathrm{H}_{2} \mathrm{O} 30 / 70$ (\%vol.) & 0.0364 & 937 & 1.83 & $54 \mathrm{deg}$ \\
Eth/ $\mathrm{H}_{2} \mathrm{O} 40 / 60$ (\%vol.) & 0.0328 & 916 & 2.11 & $42 \mathrm{deg}$ \\
\hline \hline
\end{tabular}




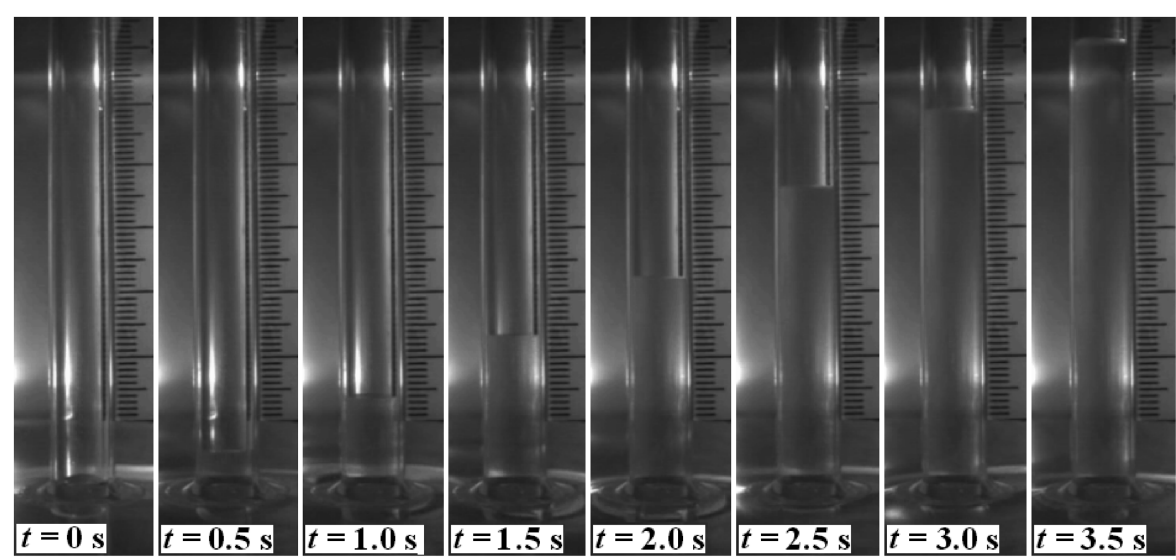

Fig. 2 Selected frames of capillary-driven flow of $\mathrm{Eth} / \mathrm{H}_{2} \mathrm{O} 30 / 70(\%$ vol.) into a tube from the experiment video under microgravity condition in the drop tower Beijing. The internal diameter $d=6 \mathrm{~mm}$ and the initial liquid height $h_{0}=10 \mathrm{~mm}$.

For the case of $\gamma_{s}=54 \mathrm{deg}$ (Fig. $4 \mathrm{~b}$ ), the velocity of the meniscus is much larger than the case of $\gamma_{s}=66 \mathrm{deg}$, which caused quite different results, as shown in Figs. $4 \mathrm{a}$ and $4 \mathrm{~b}$. From Fig. $4 \mathrm{~b}$ it can be seen that the curve entered the Lucas-Washburn flow $(\bar{h} \sim \sqrt{t})$ at microgravity time $t=2.2 \mathrm{~s}$ for $d=4 \mathrm{~mm}$, whereas for $d=6 \mathrm{~mm}$ and $d=10 \mathrm{~mm}$ the flow is still in the first region $\left(h \sim t^{2}\right)$ with positive acceleration of speed (increasing slope in the curve) until the end of the $3.6 \mathrm{~s}$ of microgravity. And the $d=4 \mathrm{~mm}$ and $d=6 \mathrm{~mm}$ curves intersect at $2.5 \mathrm{~s}$ because the velocity of the meniscus $d=$ $4 \mathrm{~mm}$ tube decreases much earlier than $d=6 \mathrm{~mm}$. However, the curve for $d=10 \mathrm{~mm}$ does not intersect with the $d=4 \mathrm{~mm}$ curve within the microgravity duration due to the lower meniscus velocity. At the end of the $3.6 \mathrm{~s}$ of microgravity, the meniscus heights in descending order are 6,4 , and $10 \mathrm{~mm}$ tubes.

In the case of $\gamma_{s}=42 \mathrm{deg}$ (Fig. $4 \mathrm{c}$ ), the velocity of the meniscus is larger than that for both cases of $\gamma_{s}=54 \mathrm{deg}$ and $\gamma_{s}=66 \mathrm{deg}$. For the same reason previously mentioned, the $d=4 \mathrm{~mm}$ curve intersects with the $d=6 \mathrm{~mm}$ curve. However, the intersection time $0.36 \mathrm{~s}$ is much earlier than that for $\gamma_{s}=54 \mathrm{deg}$. Figure $4 \mathrm{c}$ also shows that the $d=4 \mathrm{~mm}$ curve intersects with $d=10 \mathrm{~mm}$ at $2.24 \mathrm{~s}$, which is different from Fig. $4 \mathrm{~b}$. These results imply that the intersection time for the same sized tubes becomes earlier for smaller contact angles. For $\gamma_{s}=42 \mathrm{deg}$, the meniscus heights in descending order are 6,10 , and $4 \mathrm{~mm}$ tubes at the end of the $2.4 \mathrm{~s}$ microgravity.

Petrash et al. [18] performed a series of drop-tower experiments to investigate the equilibrium liquid configuration for a capillary tube in a cylindrical tank. Because their target of experiment is different from ours, only the conditions in their study in which the radius of the tubes that they chose is less than one-half the radius of the cylindrical tank are comparable to our case. Therefore, their relevant experimental results for this condition are similar to ours: the capillary liquid rise occurs in the tubes. However, the intersection of $h \sim t$ curves for different sized tubes was not observed within a quite short available microgravity time $(2.3 \mathrm{~s})$ because the relative differences of the tube sizes they chose were much larger than ours.

From Fig. 4 it can be seen that with the increase in tube size the microgravity time needed to enter Lucas-Washburn flow increased. And the $h \sim t$ curve of smaller sized tubes may intersect the curve of larger sized tubes. Therefore, the meniscus height does not decrease monotonically with the increase of the tube size within a given microgravity duration. This result can in general be explained by different behaviors of the flow corresponding to the different stages. At the initial stage of the flow process, the capillary force is dominant. For smaller sized tubes the capillary force is larger, resulting in larger meniscus velocity. When the flow enters the Lucas-Washburn region, it can be predicted from the Lucas-Washburn equation [2] that the distance of capillary rise increases with the increase of tube size. Therefore, it becomes possible for the intersection of the curves for different sized tubes. A more detailed discussion is presented in the next section.

\section{B. Discussion}

Considering the initial meniscus reorientation, the dynamic contact angle of the advancing meniscus, and the curvature of the free surface inside the container, a second-order nonlinear differential equation for the meniscus rise based on the approach of Levine et al. [6] with some improvements was put forward by Stange et al. [11]

$$
\begin{aligned}
\ddot{h} & =\frac{1}{h+h_{0}+\frac{73}{60} R}\left\{\frac{\sigma}{\rho}\left[\frac{2 s \cos \gamma_{d}}{R}-\frac{1}{R c}\right]\right. \\
& \left.-\left[\frac{8}{R^{2}}\left(h+h_{0}\right)+\frac{2}{R}\right] v \dot{h}-[1+K(x)] \frac{1}{2} \dot{h}^{2}\right\}
\end{aligned}
$$

Herein, $h$ denotes the position of the meniscus center point, and $\dot{h}$ and $\ddot{h}$ denote the meniscus velocity and acceleration, respectively. $\gamma_{d}$ is the dynamical contact angle. An incompressible, homogeneous, Newtonian liquid under isothermal conditions is assumed. The tube internal radius is $R(=1 / 2 d)$. The exponential function $s$ denotes the initial development of the meniscus from a flat to the curved equilibrium surface

$$
s(t)=1-e^{-4.6 t / t_{r}} \quad \text { with } \quad t_{r}=0.413 \sqrt{\frac{\rho R^{3}}{\sigma}}
$$

The reorientation time $t_{r}$ was proposed by Siegert et al. [19] and confirmed by the recent drop-tower experiments of Michaelis et al. [20]. The excess pressure drop $K(x)$ is a parameter that represents the velocity profile development and $K(x)=4 / 3$ in our experiments.

Equation (1) is solved numerically using a Runge-Kutta algorithm in this study. The initial conditions are $h=\dot{h}=0$ at $t=0$. The solution of Eq. (1) yields the meniscus height $h$ and the meniscus velocity $\dot{h}$ as a function of time $t$. By varying the dynamic contact angle $\gamma_{d}$ (we will not distinguish $\gamma_{d}$ and $\gamma_{s}$ for simplicity of discussion so that the contact angle will be denoted by $\gamma$ hereinafter) and tube internal radius $R$, a series of $h \sim t$ and $\dot{h} \sim t$ curves were obtained. Based on these curves the influence of the contact angle and the tube size on the capillary flow will be analyzed as follows.

The experimental results show that the meniscus height decreases monotonically with the increase in contact angle and does not decrease monotonically with the increase in tube size within a given microgravity duration. To explain the results, the numerical computations according to Eq. (1) concerning the effect of contact angle and tube size on the meniscus height and meniscus velocity of capillary rise are presented and numerical data of $h \sim t$ and $\dot{h} \sim t$ are also presented in Figs. $\underline{5}$ and 6 .

According to the experimental data in the previous paragraph, it can be seen that the duration time of region $1\left(h \sim t^{2}\right)$ and region 3 (Lucas-Washburn behavior, $h \sim \sqrt{t}$ ) are longer than region 2 $(h \sim t)$. Therefore, the contribution from the region of the velocityincreasing pattern of $\left(h \sim t^{2}\right)$ and the velocity-decreasing pattern of 


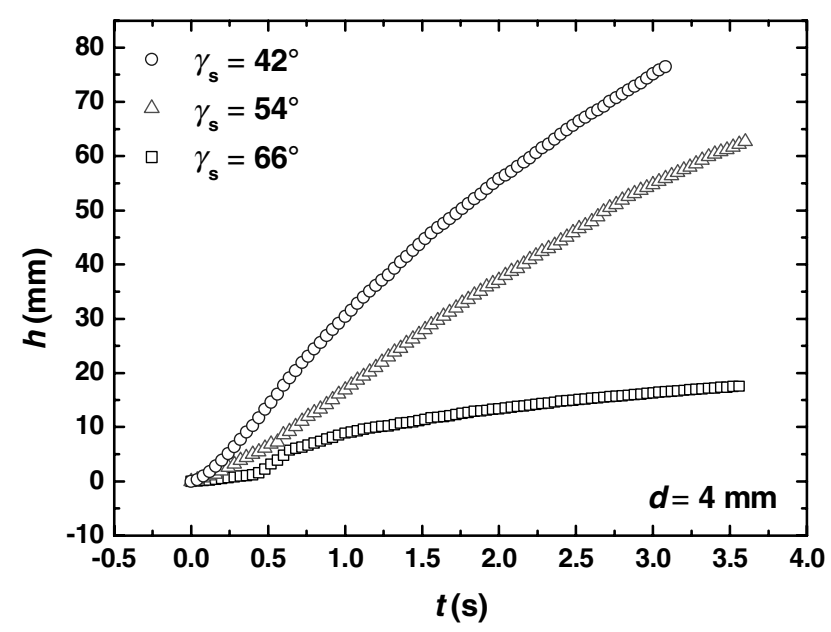

a)

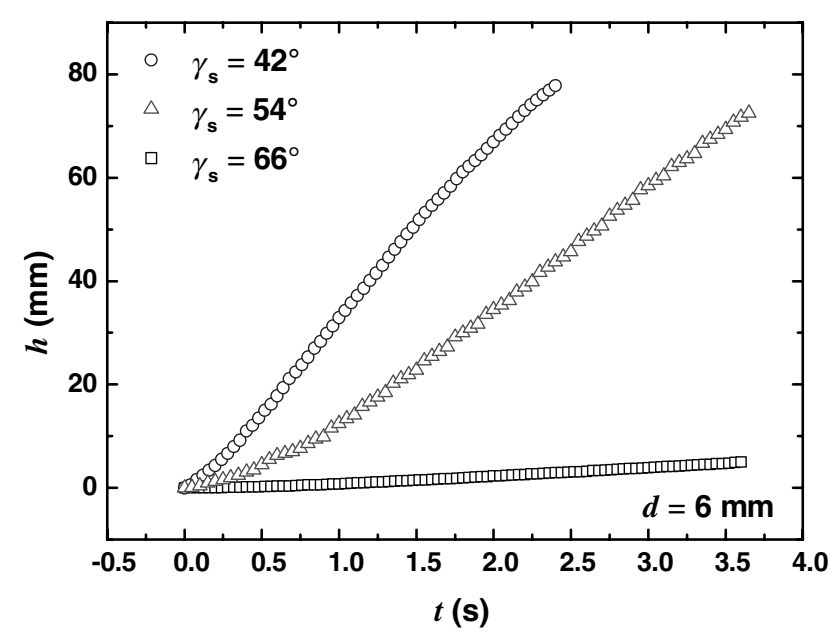

b)

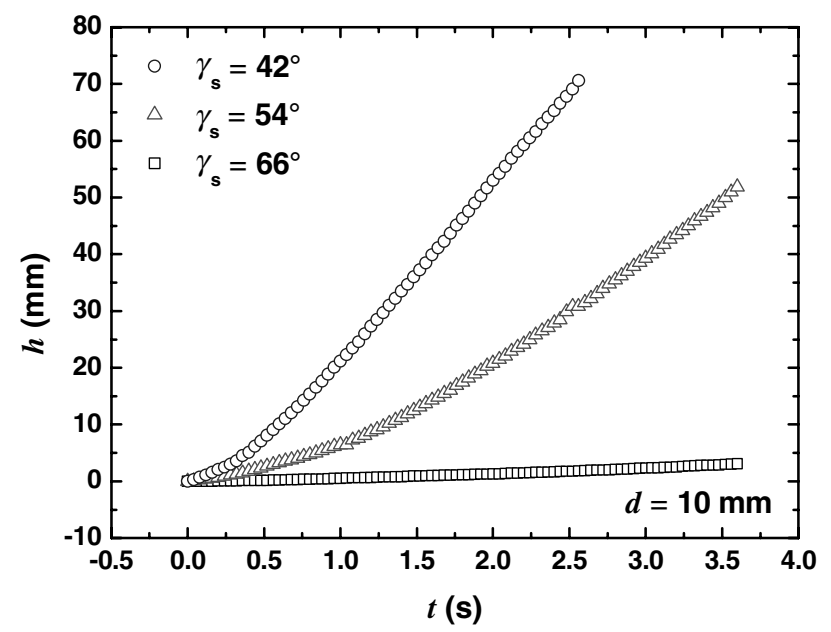

c)

Fig. 3 Experimental data from drop-tower tests. Effect of contact angle on the capillary-driven flow. Meniscus height $h$ vs time $t$ for tube diameters $d=4,6$, and $10 \mathrm{~mm}$, initial liquid heights $h_{0}=10 \mathrm{~mm}$.

( $h \sim \sqrt{t}$ ) to the meniscus height of the capillary flow within a given microgravity duration are more essential.

In Eq. (1) the denominator $h+h_{0}+(73 / 60) R$, the second, and the third items on the right-hand side describe the contributions from the inertia force, the viscous force, and convective losses, respectively. The denominator $h+h_{0}+(73 / 60) R$ plays a major

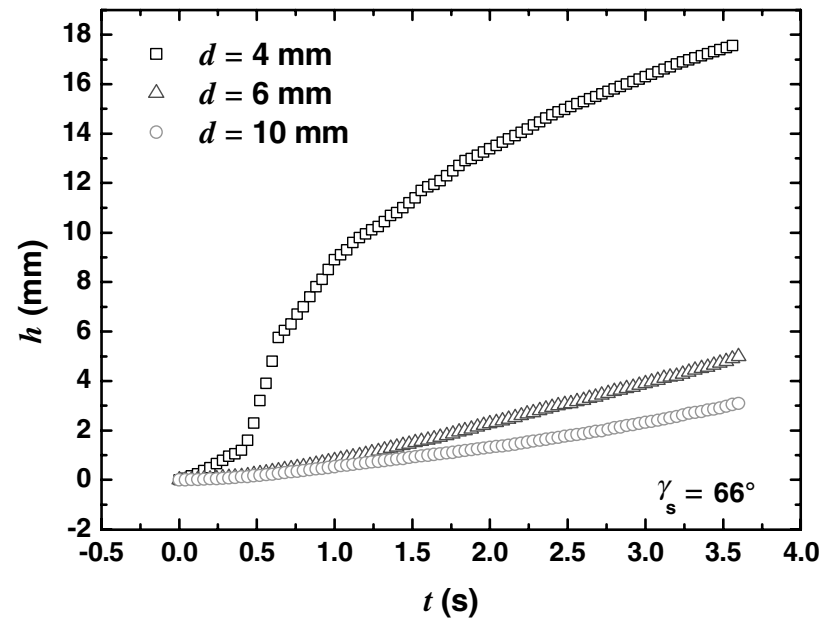

a)

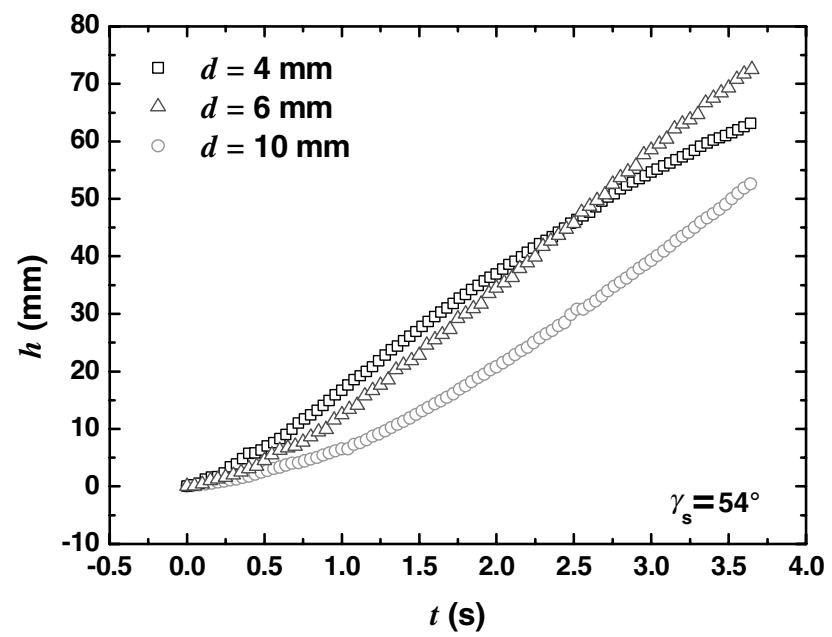

b)

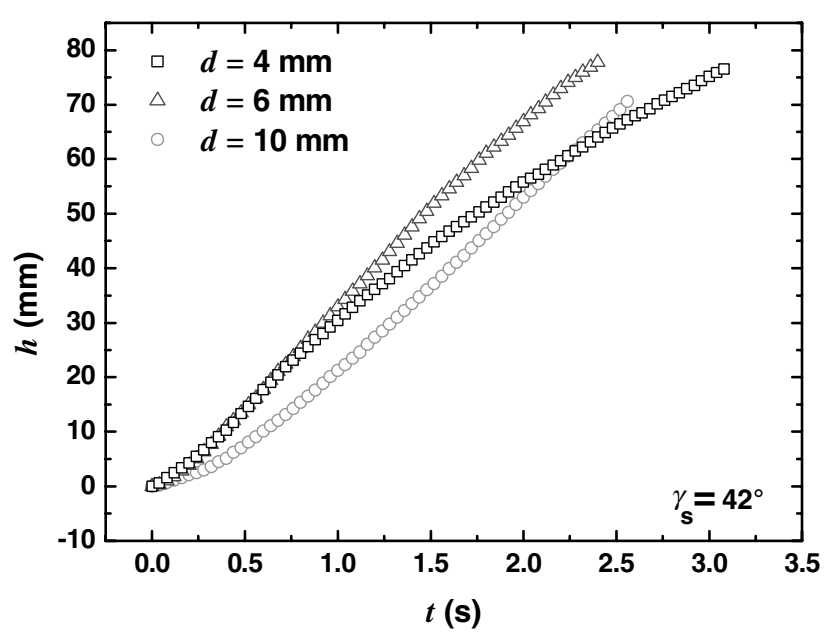

c)

Fig. 4 Experimental data from drop-tower tests. Effect of tube size on the capillary-driven flow. Meniscus height $h$ vs time $t$ for contact angle $\gamma_{s}=66,54$, and $42 \mathrm{deg}$, initial liquid heights $h_{0}=10 \mathrm{~mm}$.

role in the region of velocity increasing (region 1), and the second item plays a leading role in the region of velocity decreasing (region 3).

Figures $5 \mathrm{a}-5 \mathrm{c}$ show the meniscus height and meniscus velocity corresponding to different contact angles for $d=2 \mathrm{~mm}, d=6 \mathrm{~mm}$, and $d=20 \mathrm{~mm}$, respectively. From Fig. $\underline{5}$ we can see that with the 


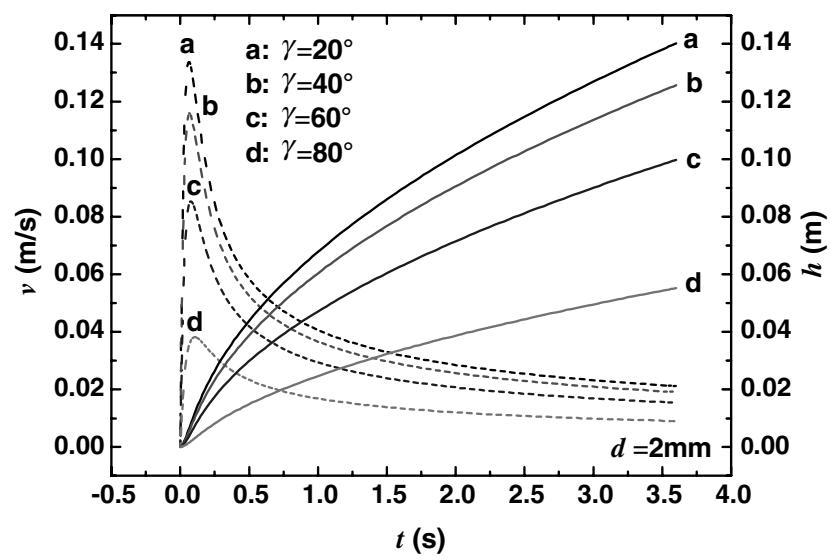

a)

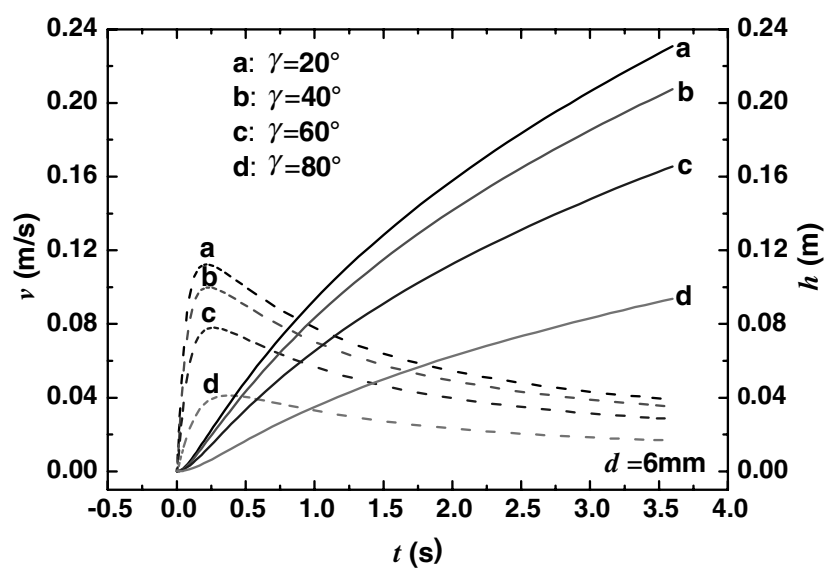

b)

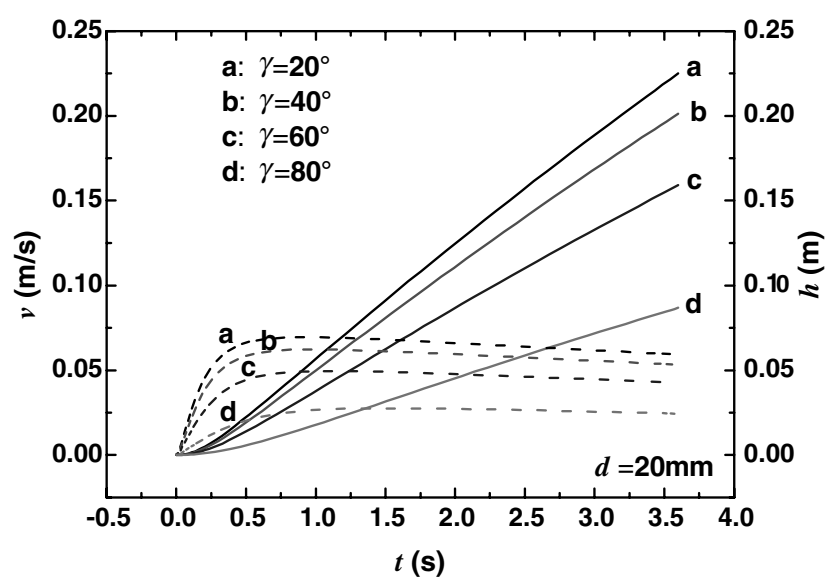

c)

Fig. 5 Numerical solutions from Runge-Kutta algorithm. Effect of contact angle on the capillary-driven flow. Meniscus height $h$ and meniscus velocity $\dot{h}$ (or $v$ ) vs time $t$ for tube diameters $d=2,6$, and $20 \mathrm{~mm}$, initial liquid heights $h_{0}=10 \mathrm{~mm}$. The dashed line refers to velocity and the solid line refers to height.

increase in contact angle the meniscus height $h$ decreased within a given microgravity duration. Because the contact angle $\gamma$, as an influence parameter on the meniscus height, appears only in the first item $(\cos \gamma)$ on the right-hand side of Eq. (1), the meniscus velocity decreases with the increase in the contact angle both in the region of velocity increasing (region 1) and velocity decreasing (region 3 ). In addition, the numerical results (see Fig. 5) show that the transition times needed to move from region 1 to region 3 for different contact angle do not change significantly. Therefore, the influences of

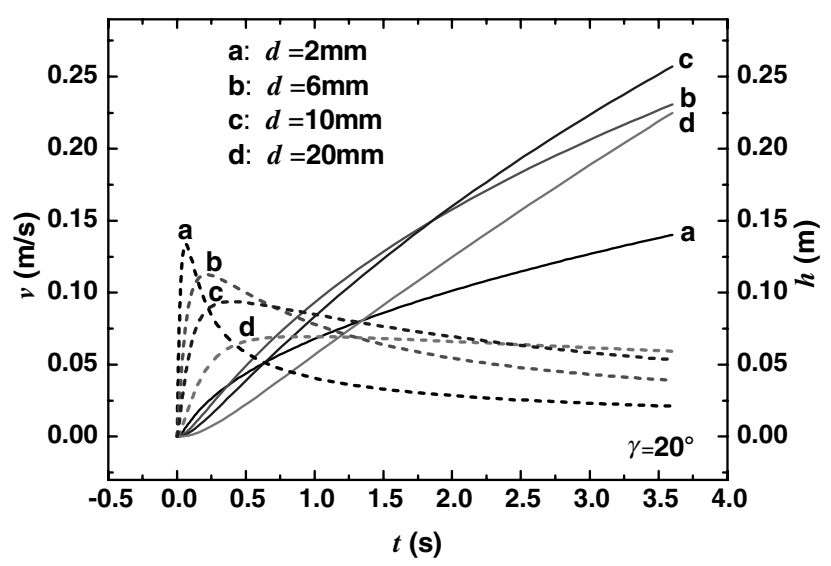

a)

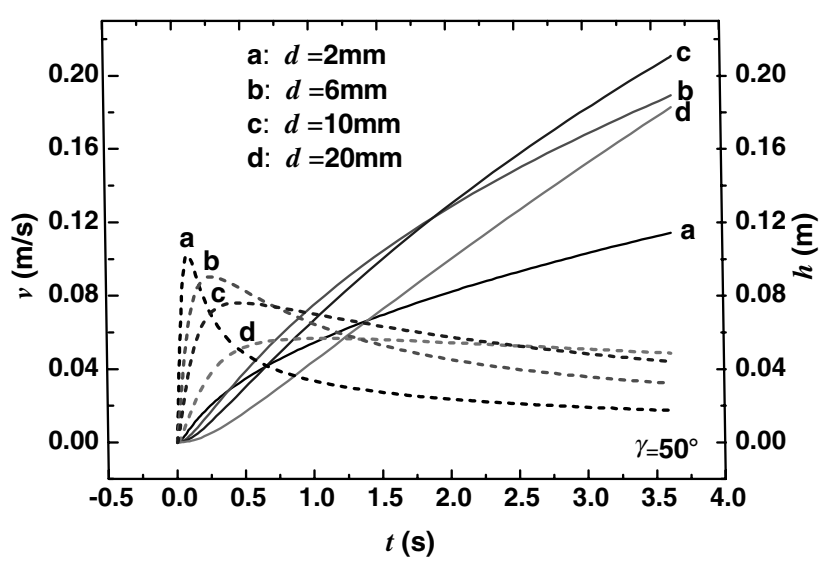

b)

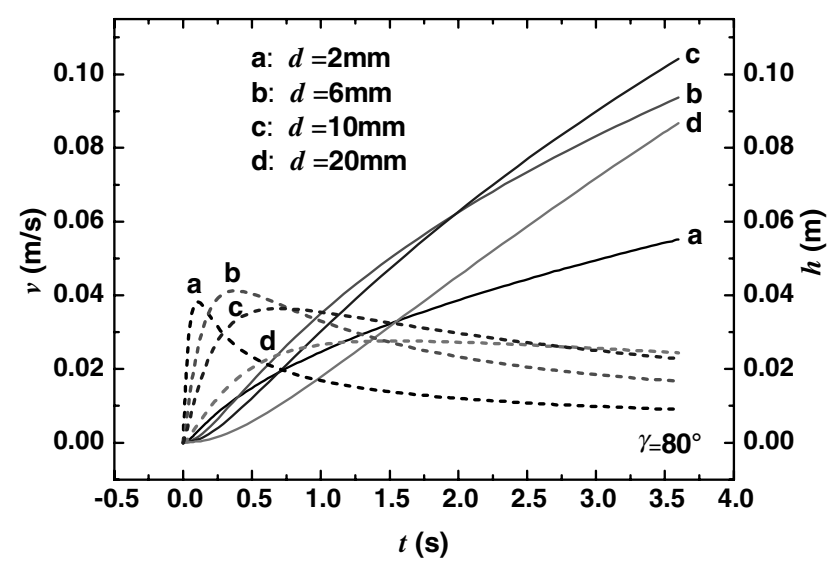

c)

Fig. 6 Numerical solutions from Runge-Kutta algorithm. Effect of tube size on the capillary-driven flow. Meniscus height $h$ and meniscus velocity $\dot{h}$ (or $v$ ) vs time $t$ for contact angle $\gamma=20 \mathrm{deg}, 50 \mathrm{deg}$, and $80 \mathrm{deg}$, initial liquid heights $h_{0}=10 \mathrm{~mm}$. The dashed line refers to velocity and the solid line refers to height.

contact angle on the meniscus velocity and meniscus height of capillary rise change monotonically.

Figures $6 a-6 c$ show the meniscus height and meniscus velocity corresponding to different tube sizes for $\gamma=20 \mathrm{deg}, \gamma=50 \mathrm{deg}$, and $\gamma=80 \mathrm{deg}$, respectively. Figure 6 shows that the meniscus height $h$ does not decrease monotonously with the increase of the tube size. For the smaller sized tube the velocity of the meniscus decreases much earlier than that of the larger sized tube. This tendency can be seen more obviously from the $\dot{h} \sim t$ curves in Fig. 6 . This behavior 
can be accounted for that the curve of the smaller sized tube may intersect the curve of the larger sized tube, as shown in the experimental results.

Alternatively, looking at Eq. (1), the parameter of the tube size $R$ exists in both the first and second item of the right-hand side of Eq. (1) and the two items have opposing contributions to the meniscus acceleration: one is positive and the other is negative. In the region of velocity increasing (region 1), the capillary force is counteracted by the inertia force. From Eq. (1) it can be expected that the acceleration of the meniscus will be larger for a smaller sized tube, which results in larger meniscus velocity. However, in the region of velocity decreasing (region 3), the flow is controlled by the friction force as shown in the second item on the right-hand side of Eq. (1). Therefore, the increase of the tube size will increase the acceleration of the meniscus so as to increase the meniscus velocity. Because of the contrary effect of the tube size on the meniscus velocity, the curves for different tube sizes could intersect each other at some point during the transition from region 1 to region 3 . In addition, the numerical results (see Fig. 6) show that with the increase of tube size, the microgravity time needed to enter the Lucas-Washburn flow zone obviously increased, which led to the intersection easier.

By comparing Figs. $6 \mathrm{a}-6 \mathrm{c}$ it can be seen that for two given tube sizes, the meniscus velocity decreases with the increase of the contact angle and therefore, the two corresponding $h \sim t$ curves intersect later. For $d=6 \mathrm{~mm}$ and $d=10 \mathrm{~mm}$, in Fig. 6 a the $h \sim t$ curves intersect at $1.8 \mathrm{~s}$, whereas in Fig. $6 \mathrm{~b}$ the $h \sim \bar{t}$ curves intersect at $1.86 \mathrm{~s}$, and in Fig. $6 \mathrm{c}$ the $h \sim t$ curves intersect at $2.0 \mathrm{~s}$ microgravity time. For $d=2 \mathrm{~mm}$ and $d=20 \mathrm{~mm}$ in Figs. 6a-6c, the $h \sim t$ curves intersect at $1.35,1.40$, and $1.55 \mathrm{~s}$ microgravity time, respectively. Therefore, in Fig. 4b the $h \sim t$ curves for $d=4 \mathrm{~mm}$ and $d=10 \mathrm{~mm}$ do not intersect within $3.6 \mathrm{~s}$ microgravity time because the contact angle is large and the meniscus velocity is slow. However, the trend that the curves of $h \sim t$ would intersect somewhere if the microgravity time can last longer is obvious.

From Eq. (1) it can be seen that the larger the meniscus velocity $\dot{h}$, the larger the friction force as shown in the second item of the righthand side. The larger friction force will make the flow enter the Lucas-Washburn region (for the same sized tubes) earlier. As mentioned previously, the intersection of curves for different sized tubes occurs during the transition from region 1 to region 3 . Therefore, the curves intersect earlier for larger velocity $\dot{h}$ for two given tube sizes. Because the meniscus velocity increases with the decrease in the contact angle for the same sized tubes, the curves intersect earlier when the contact angle is smaller. This is consistent with both experimental and numerical results from Eq. (1), as shown in Figs. 4 and 6.

Apparently, what Eq. (1) requires is the dynamic contact angle $\gamma_{d}$ to quantitatively describe the capillary flow behavior. Furthermore, the precision of the dynamic contact angle is an important factor in determining the meniscus height of capillary flow. However, how the dynamic contact angle is dependent on the meniscus velocity is still an open issue until now. As known, there are some empirical equations available for $\gamma_{d}$ in literature $[21,22]$. Unfortunately, we found that for test liquids in our study the $\gamma_{d}$ calculated using the relevant formula presented in $[21,22]$ are apparently much less than the values observed in the experiments, and therefore we do not think the two equations are suitable for our case. Considering this, static contact angles were used instead in all numerical computations in the current study. Accurate estimation of dynamic contact angles would be desirable for studying the capillary-driven flow under microgravity more quantitatively.

\section{Conclusions}

The influences of contact angle and tube size on the capillarydriven flow in circular cylindrical tubes are studied under microgravity using a $3.6 \mathrm{~s}$ drop tower. The experimental findings and related analysis can be summarized as follows:

Both the meniscus height $h$ and the velocity $\dot{h}$ decrease monotonously with microgravity time as contact angle increases. The explanation for this fact is straightforward. The contact angle is only related to the capillary force, which is a driving force for the flow. Therefore, with the increase in the contact angle (decrease in the capillary force), the meniscus velocity decreases whether it is staying in the region of velocity increasing (region 1 ) or velocity decreasing (region 3).

The $h \sim t$ curves and the $\dot{h} \sim t$ curves do not change monotonously with the increase of the tube size within some microgravity duration. The $h \sim t$ curves of different sized tubes may intersect each other. We also find that a smaller contact angle will make the intersection occur earlier. The influence of the tube size on the forces controlling the capillary flow is different from the contact angle. The larger tube size corresponds to larger volume (and mass) of liquid inside the tube. Therefore, in region 1 where the inertia force is dominant, larger tube size makes the acceleration of the flow smaller, leading to smaller velocity and height. On the other hand, in region 3 the viscous force is a dominant factor for the flow. With the increase of tube size, the tube wall will relatively have less influence on the flow so that the viscous force decreases accordingly. Thus, the increase in tube size tends to increase the flow velocity in region 3 . The contrary effect of tube size on the flow velocity in regions 1 and 3 makes it possible that $h \sim t$ curves of different sized tubes intersect each other. With the decrease in the contact angle the meniscus velocity increases and the flow enters the region of velocity decreasing earlier, which make the intersection of curves of two given sized tubes occur earlier.

The results in this study may be useful for controlling the transportation of liquid under microgravity as different sized cross sections will cause different meniscus velocities.

\section{Acknowledgments}

This work is supported by grants (numbers 10672173 and 10432060) from the National Natural Science Foundation of China and the Knowledge Innovation Program of the Chinese Academy of Sciences (including Grant No. KJCX2-YW-L08).

\section{References}

[1] Lucas, R., "Üeber das Zeitgesetz des Kapillaren Aufstiegs von Flüssigkeiten," Kolloid Zeitschrift and Zeitschrift für Polymere, Vol. 23, No. 1, 1918, pp. 15-22.

doi:10.1007/BF01461107

[2] Washburn, E. W., "The Dynamics of Capillary Flow," Physical Review, Vol. 17, No. 3, 1921, pp. 273-283. doi:10.1103/PhysRev.17.273

[3] Bell, J. M., and Cameron, F. K., "The Flow of Liquids Through Capillary Spaces," Journal of Physical Chemistry, Vol. 10, No. 8, 1906, pp. $658-674$. doi:10.1021/j150080a005

[4] Rideal, E. K., "On the Flow of Liquids Under Capillary Pressure," Philosophical Magazine, Vol. 44, No. 264, 1922, pp. 1152-1159.

[5] LeGrand, E. J., and Rense, W. A., "Data on Rate of Capillary Rise," Journal of Applied Physics, Vol. 16, No. 12, 1945, pp. 843-846. doi:10.1063/1.1707550

[6] Levine, S., Lowndes, J., Watson, E., and Neale, G. J., "A Theory of Capillary Rise of a Liquid in a Vertical Cylindrical Tube and in a Parallel-Plate Channel: Washburn Equation Modified to Account for the Meniscus with Slippage at the Contact Line," Journal of Colloid and Interface Science, Vol. 73, No. 1, 1980, pp. 136-151. doi:10.1016/0021-9797(80)90131-9

[7] Dreyer, M. E., Delgado, A., and Rath, H. J., "Capillary Rise of Liquid Between Parallel Plates Under Microgravity," Journal of Colloid and Interface Science, Vol. 163, No. 1, 1994, pp. 158-168. doi:10.1006/jcis.1994.1092

[8] Weislogel, M. M., and Lichter, S., "Capillary Flow in an Interior Corner,' Journal of Fluid Mechanics, Vol. 373, No. 1, 1998, pp. 349-378. doi:10.1017/S0022112098002535

[9] Chen, Y., and Collicott, S., "Experimental Study on Capillary Flow in a Vane-Wall Gap Geometry," AIAA Journal, Vol. 43, No. 11, 2005 , pp. 2395-2403. doi:10.2514/1.12604

[10] Concus, P., Finn, R., and Weislogel, M., "Measurement of Critical Contact Angle in a Microgravity Space Experiment," Experiments in Fluids, Vol. 28, No. 3, 2000, pp. 197-205. 
doi: $10.1007 / \mathrm{s} 003480050379$

[11] Stange, M., Dreyer, M. E., and Rath, H. J., "Capillary-Driven Flow in Circular Cylindrical Tubes," Physics of Fluids, Vol. 15, No. 9, 2003, pp. 2587-2601. doi:10.1063/1.1596913

[12] Dreyer, M. E., "Free Surface Flows Under Compensated Gravity Conditions in Springer Tracts in Modern Physics," Vol. 221, SpringerVerlag, Berlin, 2007, pp. 133-172.

[13] Dreyer, M. E., Gerstmann, J., Stange, M., Rosendahl, U., Wölk, G., and Rath, H. J., "Capillary Effects Under Low Gravity, Part 1: Surface Settling, Capillary Rise, and Critical Velocities," Space Forum, Vol. 3, 1998, pp. 87-136.

[14] Carnali, J. O., and Kotkin, C. A., "Determination of the Capillary Nature of Simple Woven Textiles," Journal of Colloid and Interface Science, Vol. 159, No. 2, 1993, pp. 319-323. doi:10.1006/jcis.1993.1329

[15] Marmur, A., and Cohen, R. D., "Characterization of Porous Media by the Kinetics of Liquid Penetration: The Vertical Capillaries Model," Journal of Colloid and Interface Science, Vol. 189, No. 2, 1997, pp. 299-304. doi:10.1006/jcis.1997.4816

[16] Weislogel, M. M., and Ross, H. D., "Surface Settling in Partially Filled Containers upon Step Reduction in Gravity," NASATM-103641, 1990.

[17] Xiaoqian, Z., Longgen, Y., Wendong, W., Lanqiao, T., and Kangzhuang, Y., "Some Key Technics of Drop-Tower Experiment Device of National Microgravity Laboratory," Science in China Series
E, Vol. 48, No. 3, 2005, pp. 305-316. doi: $10.1360 / 102004-21$

[18] Petrash, D. A., Nelson, T. M., and Otto, E. W., "Effect of Surface Energy on the Liquid-Vapor Interface Configuration During Weightlessness," NASA TN D-1582, 1963.

[19] Siegert, C. E., Petrash, D. A., and Otto, E. W., "Time Response of Liquid-Vapor Interface After Entering Weightlessness," NASA TN D2458, 1964.

[20] Michaelis, M., Dreyer, M. E., and Rath, H. J., "Experimental Investigation of the Liquid Interface Reorientation Upon Step Reduction in Gravity," Microgravity Transport Processes in Fluid, Thermal, Biological, and Materials Sciences, edited by S. S. Sadhal, Vol. 974, New York Academy of Sciences, New York, 2002, pp. 246-260.

[21] Jiang, T. S., Oh, S. G., and Slattery, J. C., "Correlation for Dynamic Contact Angle," Journal of Colloid and Interface Science, Vol. 69, No. 1, 1979, pp. 74-77. doi:10.1016/0021-9797(79)90081-X

[22] Bracke, M., Voeght, F. De, and Joos, P., "The Kinetics of Wetting: The Dynamic Contact Angle," Progress in Colloid and Polymer Science, Vol. 79, Springer-Verlag, Berlin, 1989, pp. 142-149. doi:10.1007/BFb0116200 